\title{
Modified Coulomb potential of QED in a strong magnetic field
}

\author{
Néda Sadooghi* \\ Department of Physics, Sharif University of Technology, P.O. Box 11155-9161, Tehran-Iran \\ Institute for Studies in Theoretical Physics and Mathematics (IPM), \\ School of Physics, P.O. Box 19395-9161, Tehran-Iran \\ E-mail: sadooghi@physics.sharif.ir
}

The static Coulomb potential of Quantum Electrodynamics is calculated in the presence of a strong magnetic field by computing perturbatively the vacuum expectation value of the corresponding Wilson loop in the lowest Landau level (LLL) approximation. In the LLL, two different regimes of dynamical mass, $m_{d y n}$, can be distinguished. These two regimes are $\left|\mathbf{q}_{\|}^{2}\right| \ll m_{\text {dyn. }}^{2} \ll|e B|$ and $m_{d y n .}^{2} \ll\left|\mathbf{q}_{\|}^{2}\right| \ll|e B|$, where $\mathbf{q}_{\|}$is the longitudinal components of the momentum relative to the external magnetic field $B$. As it turns out, the potential in the first regime, $\left|\mathbf{q}_{\|}^{2}\right| \ll m_{d y n}^{2}$. $\ll|e B|$, has the general form of a modfied Coulomb potential $V_{1}(R, \theta)=-\frac{\alpha}{R}\left(\mathscr{A}_{1}(\alpha, \theta)-\frac{\gamma \mathscr{S}_{2}(\alpha, \theta)}{R^{2}}+\frac{\gamma^{2} \mathscr{A}_{3}(\alpha, \theta)}{R^{4}}\right)$, where $\alpha$ is the fine structure constant and $\theta$ the angle between the particle-antiparticle axis and the external magnetic field. In the second regime, $m_{d y n .}^{2} \ll\left|\mathbf{q}_{\|}^{2}\right| \ll|e B|$, however, the potential has the general form of a modified Yukawa potential $V_{2}(R, \theta)=-\frac{\alpha e^{-M_{\text {eff }} R}}{\left(1-\frac{\alpha}{\pi}\right) g(\theta) R}$ with $M_{\text {eff. }}(\alpha, \theta) \equiv g(\theta) \sqrt{\frac{2 \alpha|e B|}{\pi}}$. The $\theta$-dependence of $V_{1}$ and $V_{2}$ is a novel property, which was not observed before in the literature. As it turns out, in the regime $\left|\mathbf{q}_{\|}^{2}\right| \ll m_{d y n}^{2}$. $\ll|e B|$, for strong enough magnetic field and depending on the angle $\theta$, a qualitative change occurs in the Coulomb-like potential $V_{1}$; Whereas for $\theta=0, \pi$ the potential is repulsive, it exhibits a minimum for angles $\theta \in] 0, \pi[$.

The XXV International Symposium on Lattice Field Theory

July 30-4 August 2007

Regensburg, Germany

\footnotetext{
*Speaker.

${ }^{\dagger}$ This contribution is based on [1], a collaboration with A.Sodeiri Jalili.
} 


\section{Introduction}

It is widely accepted that the formation of quark bound states in the QCD low energy regime, arises by spontaneous breaking of chiral symmetry of QCD Lagrangian. But, chiral symmetry breaking can also be induced dynamically in the presence of background electromagnetic fields. In particular, strong and constant magnetic fields lead to the formation of chiral symmetry breaking condensate $\langle\bar{\psi} \psi\rangle$, that plays the role of a dynamically generated fermion mass and breaks the chiral symmetry of the theory consequently. This is the well-established scenario of magnetic catalysis [2]. The essence of this effect is the dimensional reduction from $D$ to $D-2$ dimensions in the dynamics of fermion pairing in a strong magnetic field, that is believed to be dominated by the lowest Landau level (LLL).

Here, we are interested on static Coulomb potential produced by a point-like electric charge placed into a strong but constant magnetic field. Recently, this potential is calculated in [3] and [1]. In [3], it is shown that the standard Coulomb law is modified by the vacuum polarization arising in the external magnetic field. This implies a short range character of interaction, expressed as Yukawa law

$$
V(\mathbf{x})=-\frac{\alpha e^{-M_{\gamma} R}}{R}, \quad \text { with the photon mass } \quad M_{\gamma} \equiv \sqrt{\frac{2 \alpha|e B| N_{f}}{\pi}},
$$

$R \equiv|\mathbf{x}|$ the distance between a particle-antiparticle pair and $\alpha \equiv \frac{e^{2}}{4 \pi}$ the fine structure constant. In these proceedings, after briefly introducing QED in an external magnetic field, we will present the results from [1], where this potential is determined anew by computing perturbatively the vacuum expectation value (VEV) of the corresponding Wilson loop to a static fermion-antifermion pair. In the regime of LLL dominance, two different regions of dynamical mass $m_{d y n}$. $\left|\mathbf{q}_{\|}^{2}\right| \ll m_{d y n}^{2}$. $\ll|e B|$ and $m_{\text {dyn. }}^{2} \ll\left|\mathbf{q}_{\|}^{2}\right| \ll|e B|$, will be considered separately. In the regime $\left|\mathbf{q}_{\|}^{2}\right| \ll m_{d y n}^{2}$. $\ll|e B|$, the potential will be shown to have the general form of a modified Coulomb potential

$$
V_{1}(R, \theta)=-\frac{\alpha}{R}\left(\mathscr{A}_{1}(\alpha, \theta)-\frac{\gamma \mathscr{A}_{2}(\alpha, \theta)}{R^{2}}+\frac{\gamma^{2} \mathscr{A}_{3}(\alpha, \theta)}{R^{4}}\right), \quad \text { with } \quad \gamma \equiv \frac{2 \alpha}{3 \pi m_{d y n}^{2}}
$$

and in the regime $m_{d y n .}^{2} \ll\left|\mathbf{q}_{\|}^{2}\right| \ll|e B|$, it is a Yukawa-like potential

$$
V_{2}(R, \theta)=-\frac{\alpha e^{-M_{\text {eff. }} R}}{\left(1-\frac{\alpha}{\pi}\right) g(\theta) R}, \quad \text { with the photon mass } \quad M_{\text {eff. }}(\theta) \equiv g(\theta) \sqrt{\frac{2 \alpha|e B|}{\pi}} .
$$

In (1.2) and (1.3), $\mathscr{A}_{i}, i=1,2,3$ and $g(\theta)$, whose exact expressions will be presented in Section 4 , are some functions depending on $\theta$, the angle between the particle-antiparticle axis and the direction of the magnetic field. Up to this explicit novel dependence on $\theta$, the potential $V_{2}(R, \theta)$ from (1.3) is comparable with the potential (1.1) from [3]. The consequences of the $\theta$ dependence will be also discussed in Section 4.

\section{QED in a strong magnetic field}

We start with the QED Lagrangian density

$$
\mathscr{L}=-\frac{1}{4} F_{\mu v} F^{\mu v}+\bar{\psi} \gamma^{\mu}\left(i \partial_{\mu}+e A_{\mu}\right) \psi-m \bar{\psi} \psi,
$$


where the vector field $A_{\mu}=a_{\mu}+A_{\mu}^{\text {ext. }}$. Here, $a_{\mu}$ is the Abelian quantum field and $F_{\mu \nu}$ is the corresponding field strength. $A_{\mu}^{\text {ext. }}$ describes an external electromagnetic field. It is possible to fix the gauge so that the magnetic field is directed in the third direction. Here, we choose the symmetric gauge, $A_{\mu}^{\text {ext. }}=\frac{B}{2}\left(0, x_{2},-x_{1}, 0\right)$. Using the Schwinger proper time formalism [4], the full photon propagator in the LLL approximation is given by [2]

$$
i \widetilde{\mathscr{D}}_{\mu v}(q)=\frac{g_{\mu \nu}^{\|}}{q^{2}+\mathbf{q}_{\|}^{2} \Pi\left(\mathbf{q}_{\|}^{2}, \mathbf{q}_{\perp}^{2}\right)},
$$

with $\Pi\left(q_{\perp}^{2}, q_{\|}^{2}\right)$ having the form [5]

$$
\begin{array}{ccc}
\Pi\left(\mathbf{q}_{\perp}^{2}, \mathbf{q}_{\|}^{2}\right) \simeq+\frac{\alpha|e B| N_{f}}{3 \pi m_{d y n}^{2}} e^{-\frac{q_{\perp}^{2}}{2|e B|}} \quad \text { for } & \left|\mathbf{q}_{\|}^{2}\right| \ll m_{d y n .}^{2} \ll|e B|, \\
\Pi\left(\mathbf{q}_{\perp}^{2}, \mathbf{q}_{\|}^{2}\right) \simeq-\frac{2 \alpha|e B| N_{f}}{\pi \mathbf{q}_{\|}^{2}} e^{-\frac{q_{\perp}^{2}}{2|e B|}} & \text { for } & m_{\text {dyn. }}^{2} \ll\left|\mathbf{q}_{\|}^{2}\right| \ll|e B| .
\end{array}
$$

In the second regime, the photons acquire a finite mass. This can be shown by plugging (2.4) in the full photon propagator (2.2) and assuming that $\left|\mathbf{q}_{\perp}^{2}\right| \ll|e B|$. The corresponding photon propagator is then given by

$$
\widetilde{\mathscr{D}}_{\mu v}(q) \approx-\frac{i g_{\mu \nu}^{\|}}{q^{2}-M_{\gamma}^{2}}, \quad \text { with the photon mass } \quad M_{\gamma}=\sqrt{\frac{2 \alpha|e B| N_{f}}{\pi}} .
$$

The appearance of a finite photon mass $M_{\gamma}$ is the result of the dimensional reduction $3+1 \rightarrow 1+1$ in the presence of a constant magnetic field. As for the dynamically generated fermion mass $m_{d y n}$., it can be determined nonperturbatively by solving the corresponding Schwinger-Dyson equation in the rainbow (ladder) approximation [2]. It is given by

$$
m_{d y n .}=C \sqrt{e B} \exp \left(-\frac{\pi}{2}\left(\frac{\pi}{2 \alpha}\right)^{1 / 2}\right),
$$

where the constant $C$ is of order one.

\section{The Wilson loop and the modified Coulomb potential in a strong magnetic field}

In ordinary QED, the static Coulomb potential $V(R)$ between a particle and an antiparticle is given by the VEV of a closed Wilson loop (see [6] and the references therein)

$$
V(R)=-\lim _{T \rightarrow \infty} \frac{1}{T} \ln \left\langle W_{C}[A]\right\rangle,
$$

where the loop correlation function - the Wilson loop, is defined by

$$
W_{C}[A] \equiv e^{i e \oint_{C} A_{\mu}(x) d x_{\mu}} .
$$

The VEV in (3.1) is given by

$$
\langle\mathscr{O}\rangle \equiv \frac{\int \mathscr{D} A_{\mu} \mathscr{D} \psi \mathscr{D} \bar{\psi} \mathscr{O} e^{-S}}{\int \mathscr{D} A_{\mu} \mathscr{D} \psi \mathscr{D} \bar{\psi} e^{-S}}
$$


For $S$ being the QED action, the Coulomb potential $V(R)=-\frac{e^{2}}{4 \pi R}$ can be analytically calculated in the quenched approximation. To do this, we expand $\left\langle W_{C}[A]\right\rangle$ in powers of the background field $A_{\mu}$

$$
\left\langle W_{C}[A]\right\rangle=\left\langle 1+i e \oint_{C} d x_{\mu} A_{\mu}(x)-\frac{e^{2}}{2} \oint_{C} \oint_{C} d x_{\mu} d y_{v} A_{\mu}(x) A_{v}(y)+\cdots\right\rangle .
$$

Plugging (3.4) in (3.1), the potential reads

$$
V(R)=\lim _{T \rightarrow \infty} \frac{e^{2}}{2 T} \oint_{C} \oint_{C} d x_{\mu} d y_{v} D^{\mu v}(x, y)+\mathscr{O}\left(e^{3}\right) .
$$

Replacing now $D^{\mu v}(x, y)$ by the photon propagator of the ordinary QED in the coordinate space $D_{\mu v}(x, y)=\frac{\delta_{\mu v}}{4 \pi^{2}(x-y)^{2}}$, the standard Coulomb potential can be derived. Note that, in (3.4), the second term including only one gauge field does not contribute. As for the other terms, those with an odd number of external photon lines does not contribute to the above expansion. This is because of the Furry's theorem, that holds in ordinary QED in contrary to QED in the presence of external magnetic field. In the next section, using the above idea the particle-antiparticle potential in the LLL approximation will be derived in two different regimes by replacing $D^{\mu v}(x, y)$ in (3.5) with the full photon propagator from (2.2)-(2.4).

\section{Results}

\subsection{Modified Coulomb potential in $\left|\mathbf{q}_{\|}^{2}\right| \ll m_{\text {dyn. }}^{2} \ll|e B|$ regime}

Substituting (2.3) with $N_{f}=1$ in (2.2) the full photon propagator in the coordinate space in this regime is given by

$$
\widetilde{\mathscr{D}}_{\mu \nu}(x)=-i g_{\mu \nu}^{\|} \int \frac{d^{4} q}{(2 \pi)^{4}} \frac{e^{i q x}}{q^{2}+\frac{\alpha|e B|}{3 \pi m_{d y n}^{2}} \mathbf{q}_{\|}^{2} \exp \left(-\frac{\mathbf{q}_{\perp}^{2}}{2|e B|}\right)} .
$$

After a lengthy but straightforward calculation, we arrive at (see [1] for more details)

$$
\begin{aligned}
& \widetilde{\mathscr{D}}_{\mu \nu}(R, \theta, T)=\frac{\delta_{\mu \nu}^{\|}}{4 \pi^{2} a_{1}}\left[\left(1+\frac{\gamma}{a_{1}}-\frac{4 a_{2}+\gamma R^{2} \sin ^{2} \theta}{2 \beta a_{1}^{2}}+\frac{3 a_{2} R^{2} \sin ^{2} \theta}{2 \beta^{2} a_{1}^{3}}\right)\right. \\
& +\frac{4 \gamma^{2}}{a_{1}^{2}}\left(1-\frac{3 R^{2} \sin ^{2} \theta}{2 \beta a_{1}}+\frac{3 R^{4} \sin ^{4} \theta}{8 \beta^{2} a_{1}^{2}}\right) \\
& -\frac{12 \gamma a_{2}}{\beta a_{1}^{3}}\left(2-\frac{2}{\beta a_{1}}\left(2 R^{2} \sin ^{2} \theta+\frac{a_{2}}{\gamma}\right)+\frac{5}{4 \beta^{2} a_{1}^{2}}\left(R^{4} \sin ^{4} \theta+\frac{4 a_{2} R^{2} \sin ^{2} \theta}{\gamma}\right)-\frac{15 a_{2} R^{4} \sin ^{4} \theta}{8 \gamma \beta^{3} a_{1}^{3}}\right) \\
& \left.-\frac{\gamma}{|e B| \beta a_{1}^{2}}\left(1-\frac{3}{\beta a_{1}}\left(\frac{R^{2} \sin ^{2} \theta}{2}+\frac{a_{2}}{\gamma}\right)+\frac{6}{\beta^{2} a_{1}^{2}}\left(\frac{R^{4} \sin ^{4} \theta}{16}+\frac{a_{2} R^{2} \sin ^{2} \theta}{\gamma}\right)-\frac{15 a_{2} R^{4} \sin ^{4} \theta}{8 \gamma \beta^{3} a_{1}^{3}}\right)\right] .
\end{aligned}
$$

Here, $\beta^{-1} \equiv 4\left(1+\frac{\alpha|e B|}{3 \pi m_{d y n .}^{2}}\right)$ and $\gamma(\alpha) \equiv \frac{2 \alpha}{3 \pi m_{d y n .}^{2}}$, are constant c-numbers, and $a_{1,2}=a_{1,2}(R, \theta, T)$ are defined by $a_{1}(R, \theta, T) \equiv T^{2}+R^{2} f^{2}(\alpha, \theta)$ with $f^{2}(\alpha, \theta) \equiv 1+\frac{\gamma|e B|}{2} \sin ^{2} \theta$, and $a_{2}(R, \theta, T) \equiv$ $\beta \gamma\left(T^{2}+R^{2} \cos ^{2} \theta\right)$. In all these expressions $R \equiv|\mathbf{x}|$ is the distance between the static fermion 
and antifermion pair, $\theta$ is the angle between the particle-antiparticle axis and the direction of the magnetic field $B$, i.e. the $x_{3}$ direction, and $T \equiv i x_{0}$ is the Euclidean time. The static Coulomb potential can then be calculated using (3.5) and reads

$$
V(R, \theta)=-\frac{\alpha}{R}\left(\mathscr{A}_{1}(\alpha, \theta)-\frac{\gamma \mathscr{A}_{2}(\alpha, \theta)}{R^{2}}+\frac{\gamma^{2} \mathscr{A}_{3}(\alpha, \theta)}{R^{4}}\right)
$$

with

$$
\begin{aligned}
& \mathscr{A}_{1}(\alpha, \theta) \equiv \frac{1}{f}, \\
& \mathscr{A}_{2}(\alpha, \theta) \equiv-\frac{1}{4 f^{3}}\left(1-\frac{3 \cos ^{2} \theta}{f^{2}}-\frac{3 \sin ^{2} \theta}{8 \beta f^{2}}+\frac{15 \sin ^{2} \theta \cos ^{2} \theta}{8 \beta f^{4}}\right), \\
& \mathscr{A}_{3}(\alpha, \theta) \equiv+\frac{9}{16 f^{5}}\left(1-\frac{5 \sin ^{2} \theta}{4 \beta f^{2}}+\frac{35 \sin ^{4} \theta}{128 \beta^{2} f^{4}}\right) \\
& -\frac{15 \cos ^{2} \theta}{8 f^{7}}\left(3-\frac{7}{4 \beta f^{2}}\left(2 \beta \cos ^{2} \theta+3 \sin ^{2} \theta\right)+\frac{63 \sin ^{2} \theta}{8 \beta^{2} f^{4}}\left(\beta \cos ^{2} \theta+\frac{3 \sin ^{2} \theta}{16}\right)-\frac{693 \cos ^{2} \theta \sin ^{4} \theta}{256 \beta^{2} f^{6}}\right) \\
& -\frac{3}{16|e B| f^{5} \gamma \beta}\left(1-\frac{5}{4 \beta f^{2}}\left(4 \beta \cos ^{2} \theta+\sin ^{2} \theta\right)+\frac{35 \sin ^{2} \theta}{4 \beta^{2} f^{4}}\left(\beta \cos ^{2} \theta+\frac{\sin ^{2} \theta}{32}\right)-\frac{315 \cos ^{2} \theta \sin ^{4} \theta}{128 \beta^{2} f^{6}}\right) .
\end{aligned}
$$

Figure 1 shows this potential for different choices of the magnetic field $B=10^{5}, 10^{6}, 10^{7}, 10^{9}$ (from right to left) and different $\theta=0, \pi / 3,2 \pi / 3, \pi$. According to this result, for $R \rightarrow 0$ the potential falls more rapidly to $-\infty$ the smaller the magnetic field is and the shape of the potentials are almost the same for different choices of $\theta$.

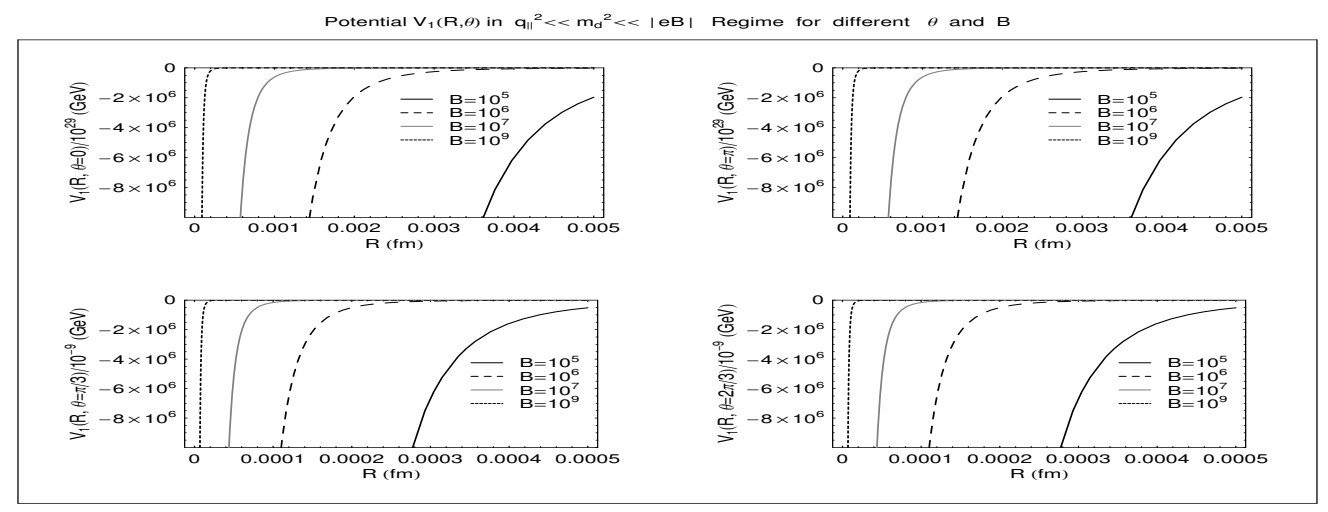

Figure 1: Potential $V_{1}(R, \theta)$ for different $B$ and $\theta$. No qualitative changes occurs by varying the angle $\theta$.

This situation changes by neglecting the coefficient $\gamma^{2} \mathscr{A}_{3}$ comparing with $\mathscr{A}_{1}$ and $\gamma_{\mathscr{A}_{2}}$ in $V_{1}(R, \theta)$. In [1] we have studied the behavior of the coefficients $\mathscr{A}_{1}, \gamma \mathscr{A}_{2}$ and $\gamma^{2} \mathscr{A}_{3}$ as a function of the angle $\theta$ for different magnetic fields. As it turns out, the coefficients $\mathscr{A}_{1}, \gamma_{\mathscr{A}_{2}}$ and $\gamma^{2} \mathscr{A}_{3}$ are positive $\forall \theta \in[0, \pi]$ and for any choice of constant magnetic field $B$, but $\mathscr{A}_{3}$ falls down rapidly by increasing the strength of the magnetic field and can be indeed neglected. For $B \geq 10^{5}$, the potential $V_{1}(R, \theta)$ can be therefore replaced by $V_{3}(R, \theta)=-\frac{\alpha}{R}\left(\mathscr{A}_{1}(\alpha, \theta)-\frac{\gamma \mathscr{A}_{2}(\alpha, \theta)}{R^{2}}\right)$, which has its minimum at 
$R_{\min }(B, \theta)=\sqrt{\frac{3 \gamma \mathscr{L}_{2}}{\mathscr{A}_{1}}}$. Figure 2 shows $V_{3}(R, \theta)$ for different choices of the magnetic field $B=$ $10^{5}, 10^{6}, 10^{7}$ and $10^{9}$ (from right to left) and different $\theta=0, \pi / 3, \pi / 2,2 \pi / 3, \pi$. As it turns out, whereas for $\theta=0, \pi$ the potential is repulsive, it exhibits a minimum for angles $\theta \in] 0, \pi[$ and distances $R \leq 0.005 \mathrm{fm}$. Moreover, the depth of the potential at $R_{\min }$ increases with the magnetic field. We interpret this effect as a possibility for bound state formation. The answer to the question concerning the existence and the number of bound states in the potential $V_{3}$ is beyond the scope of this work ${ }^{1}$.

Potential $\mathrm{V}_{3}(\mathrm{R}, \theta)$ in $\mathrm{q}_{\|}{ }^{2}<<\mathrm{m}_{\mathrm{d}}{ }^{2}<<|\mathrm{eB}|$ Regime for different $\theta$ and $\mathrm{B}$

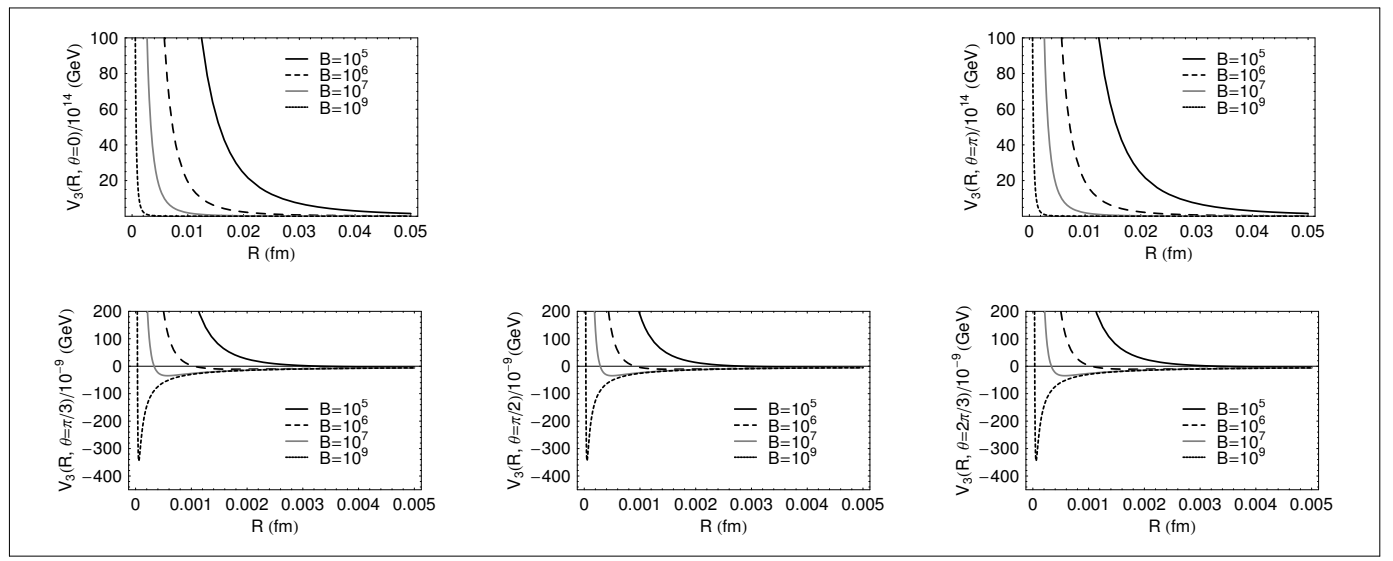

Figure 2: Potential $V_{3}(R, \theta)$ for different $B$ and $\theta$. Bound states can be formed for $\left.\theta \in\right] 0, \pi[$ and for strong magnetic fields $B \geq 10^{5}$ in the regime $R \leq 0.005 \mathrm{fm}$. The depth of the potential at $R_{\min }$ increases with the magnetic field.

\subsection{Modified Coulomb potential in $m_{d y n}^{2} \ll\left|\mathbf{q}_{\|}^{2}\right| \ll|e B|$ regime}

To compute the inter-particle potential in this regime, we have to determine first the photon propagator in the coordinate space. To do this, we substitute (2.4) in (2.2). For $N_{f}=1$, we arrive first at

$$
\widetilde{\mathscr{D}}_{\mu \nu}(x)=-i g_{\mu \nu}^{\|} \int \frac{d^{4} q}{(2 \pi)^{4}} \frac{e^{i q x}}{q^{2}-\frac{2 \alpha|e B|}{\pi} \exp \left(-\frac{\mathbf{q}_{\perp}^{2}}{2|e B|}\right)} .
$$

The integration over $q$ can be performed using the approximation $\mathbf{q}_{\perp}^{2} \ll|e B|$ which is valid in the regime of LLL dominance (see [1] for more details). We arrive at

$$
\widetilde{\mathscr{D}}_{\mu \nu}(R, \theta, T)=\frac{\delta_{\mu \nu}^{\|}}{4 \pi^{2}\left(1-\frac{\alpha}{\pi}\right)} \frac{M_{\gamma}}{\sqrt{T^{2}+R^{2} g^{2}(\theta)}} K_{1}\left(M_{\gamma} \sqrt{T^{2}+R^{2} g^{2}(\theta)}\right),
$$

where $M_{\gamma} \equiv \sqrt{\frac{2 \alpha|e B|}{\pi}}$, and $g(\theta) \equiv \sqrt{\cos ^{2} \theta+\frac{\sin ^{2} \theta}{1-\frac{\alpha}{\pi}}}$. Using now (3.5), the modified potential can be determined and reads

$$
V(R, \theta)=-\frac{\alpha}{\left(1-\frac{\alpha}{\pi}\right) g(\theta) R} e^{-M_{\gamma} g(\theta) R},
$$

\footnotetext{
${ }^{1}$ A nonperturbative analysis of the corresponding Schrödinger equation describing the Nambu-Goldstone modes and arising from a Bethe-Salpeter equation for bound states shows that at least one bound state can be formed in the attractive potential in $D=4$ dimensions [2].
} 
This potential is indeed comparable with the attractive Yukawa potential $V_{\text {Yukawa }}(R)=-\frac{\alpha}{R} e^{-m R}$, with $\alpha \rightarrow \frac{\alpha}{\left(1-\frac{\alpha}{\pi}\right) g(\theta)}$ and the effective photon mass $m \rightarrow M_{\text {eff. }}(\theta) \equiv M_{\gamma} g(\theta)$.

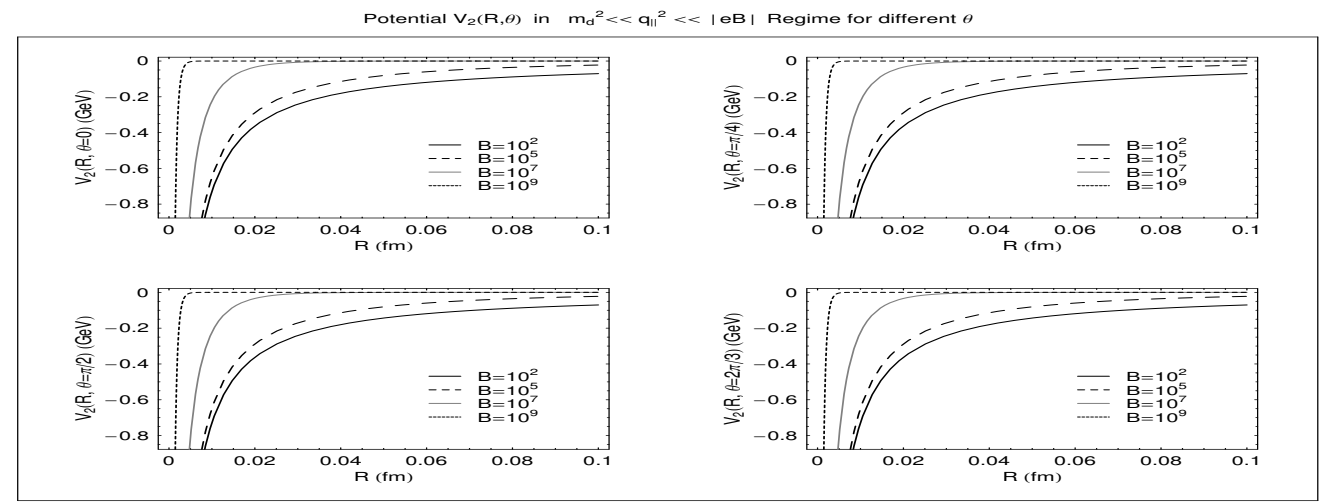

Figure 3: Potential $V_{2}(R, \theta)$ for different $B$ and $\theta$. No qualitative changes occurs by varying the angle $\theta$.

This result is in agreement with the general result about the massive photons in a strong magnetic field in the LLL approximation. It is well-known that in the regime $m_{d y n}^{2} \ll\left|\mathbf{q}_{\|}^{2}\right| \ll|e B|$, the $3+1$ dimensional QED in the LLL approximation is reduced to a $1+1$ dimensional Schwinger model, where the photon acquires a finite mass $M_{\gamma}$. Comparing to the above result, the effective photon mass in this regime is given by $M_{\text {eff. }}=M_{\gamma} g(\theta)$ and depends explicitly on the angle $\theta$ between the particle axis and the direction of the external magnetic field. Figure 3 shows the potential $V_{2}(R, \theta)$ in the second regime $m_{d y n}^{2}$. $\ll\left|\mathbf{q}_{\|}^{2}\right| \ll|e B|$ of LLL, for different magnetic field $B$ and angle $\theta$. Again no qualitative changes occurs by varying the angle $\theta$. It would be interesting to determine the inter-particle potential of QED in the LLL using a nonperturbative lattice calculation of the corresponding Wilson loop.

\section{References}

For a complete list of references see [1].

[1] N. Sadooghi and A. Sodeiri Jalili, New look at the modified Coulomb potential, Phys. Rev. D. 76, 065013 (2007).

[2] V. P. Gusynin, V. A. Miransky and I. A. Shovkovy, Dynamical chiral symmetry breaking by a magnetic field in QED, Phys. Rev. D 52, 4747 (1995).

[3] A. E. Shabad and V. V. Usov, Modified Coulomb Law in a Strongly Magnetized Vacuum, Phys. Rev. Lett. 98, 180403 (2007); ibid. Electric field of a point-like charge in a strong magnetic field, arXiv:astro-ph/0607499.

[4] J. S. Schwinger, On gauge invariance and vacuum polarization, Phys. Rev. 82, 664 (1951).

[5] G. Calucci and R. Ragazzon, Nonlogarithmic terms in the strong field dependence of the photon propagator, J. Phys. A 27, 2161 (1994); A. V. Kuznetsov and N. V. Mikheev, Electron mass operator in a strong magnetic field and dynamical chiral symmetry breaking, Phys. Rev. Lett. 89, 011601 (2002).

[6] J. B. Kogut, A review of the lattice gauge theory approach to Quantum Chromodynamics, Rev. Mod. Phys. 55, 775 (1983). 